\title{
Host-response biomarkers for diagnosis of late-onset septicemia and necrotizing enterocolitis in preterm infants
}

\author{
Pak Cheung Ng,1,2 Irene Ling Ang,, ${ }^{1,3}$ Rossa Wai Kwun Chiu,,1,4 Karen Li, 1,2 Hugh Simon Lam,2 \\ Raymond Pui On Wong, ${ }^{2}$ Kit Man Chui, ${ }^{2}$ Hon Ming Cheung, ${ }^{2}$ Eddy Wing Yin Ng, ${ }^{1,3}$ Tai Fai Fok, $^{2}$ \\ Joseph Jao Yiu Sung, 1,3 Yuk Ming Dennis Lo, 1,4 and Terence Chuen Wai Poon¹,3 \\ ${ }^{1}$ Li Ka Shing Institute of Health Sciences, ${ }^{2}$ Department of Pediatrics, ${ }^{3}$ Department of Medicine and Therapeutics, and \\ ${ }^{4}$ Department of Chemical Pathology, Prince of Wales Hospital, The Chinese University of Hong Kong, Hong Kong.
}

\begin{abstract}
Preterm infants are highly susceptible to life-threatening infections that are clinically difficult to detect, such as late-onset septicemia and necrotizing enterocolitis (NEC). Here, we used a proteomic approach to identify biomarkers for diagnosis of these devastating conditions. In a case-control study comprising 77 sepsis/NEC and 77 nonsepsis cases (10 in each group being monitored longitudinally), plasma samples collected at clinical presentation were assessed in the biomarker discovery and independent validation phases. We validated the discovered biomarkers in a prospective cohort study with 104 consecutively suspected sepsis/NEC episodes. Proapolipoprotein CII (Pro-apoC2) and a des-arginine variant of serum amyloid A (SAA) were identified as the most promising biomarkers. The ApoSAA score computed from plasma apoC2 and SAA concentrations was effective in identifying sepsis/NEC cases in the case-control and cohort studies. Stratification of infants into different risk categories by the ApoSAA score enabled neonatologists to withhold treatment in $45 \%$ and enact early stoppage of antibiotics in $16 \%$ of nonsepsis infants. The negative predictive value of this antibiotic policy was $100 \%$. The ApoSAA score could potentially allow early and accurate diagnosis of sepsis/NEC. Upon confirmation by further multicenter trials, the score would facilitate rational prescription of antibiotics and target infants who require urgent treatment.
\end{abstract}

\section{Introduction}

Advances in neonatal intensive care have led to substantial improvement in survival of preterm, very low birth weight (VLBW) $(<1,500 \mathrm{~g})$ infants. However, preterm infants are prone to opportunistic and life-threatening infections and necrotizing enterocolitis (NEC) (1-3). A recent multicenter survey suggests that up to $21 \%$ of VLBW infants encounter at least one episode of late-onset (>72 hours of life) blood culture-proven sepsis (3). Late-onset neonatal infection and NEC are associated with serious morbidity, including bronchopulmonary dysplasia (4), short bowel syndrome (5), parenteral nutrition-associated liver disease (6), and adverse neurodevelopmental outcomes $(7,8)$ in addition to increased mortality (3). Yet early warning signs and symptoms are frequently nonspecific and inconspicuous and can easily be mistaken as due to noninfected etiologies, such as exacerbations of bronchopulmonary dysplasia, apnea of prematurity, gastroesophageal reflux, or functional intestinal dysmotility (9-11). More alarmingly, the clinical deterioration may progress in a fulminant manner resulting in shock, disseminated intravascular coagulation (DIC), and death within hours of clinical presentation (12). The effectiveness of blood culture as the "gold standard" for diagnosing septicemia is, however, compromised in this group of preterm infants. A positive culture may take more than 48 hours of incubation, and false negative results are common due to the small volume of blood sample available and the intermittent presence and low density of

Authorship note: Pak Cheung Ng and Irene Ling Ang contributed equally to this work.

Conflict of interest: The authors have declared that no conflict of interest exists. Citation for this article: J Clin Invest. 2010;120(8):2989-3000. doi:10.1172/JCI40196. circulating bacteria at the early stage of infection. Thus, the discovery of novel biomarkers for early and accurate identification of septicemia and NEC from suspected cases could help to prevent unnecessary use of antibiotics in nonsepsis/non-NEC infants. The dilemma of whether to start antibiotics immediately after sepsis screening and when to stop treatment remains a major diagnostic challenge in neonatology $(10,13)$.

Neonatal septicemia and NEC are associated with hyperinflammatory host responses. Much research in the past decade has been focused on the exploration of 1 or a panel of inflammatory mediators for diagnosis of systemic infection and NEC in preterm infants $(10,13)$. All these studies used the "candidate marker" approach for selecting specific mediators in the inflammatory cascade or cellsurface antigens that are either up- or downregulated in response to pathogen invasion $(10,13)$. Although the mediators are purposefully chosen because of their favorable biochemical properties and crucial roles in infection or inflammation, this approach has the disadvantage of limiting the search of potential markers to only known proteins. Mass spectrometry-based proteomic profiling technologies, such as ProteinChip surface-enhanced laser desorption/ionization (SELDI), have been used to identify host-response signature proteins for diagnosis of many diseases and pathologic conditions, such as early-onset neonatal sepsis, intra-amniotic inflammation, and severe acute respiratory syndrome (14-17).

The objective of this study was to apply an unbiased approach to identify novel host-response biomarkers for early and accurate diagnosis of septicemia and/or NEC in preterm infants. We also aimed to develop a new clinical strategy for starting and early stoppage of antibiotic treatment in different risk categories of infants. The mass spectrometry-based proteomic profiling technology was 


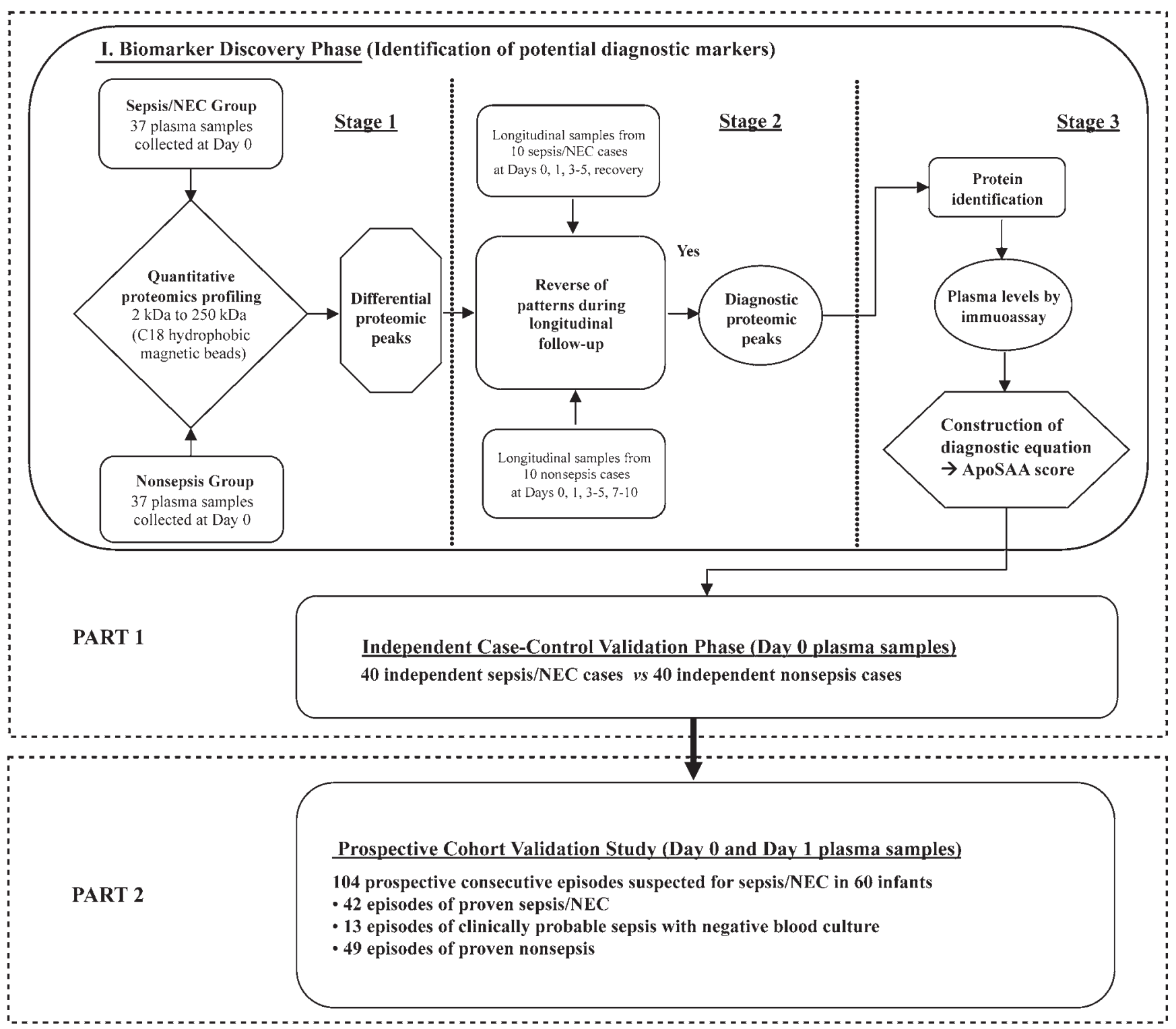

Figure 1

The study design (part 1: discovery and independent validation; part 2: prospective validation) and patient flow for discovery and validation of the biomarkers for diagnosis of sepsis and NEC in preterm neonates.

used to identify differential host-response proteins in the plasma samples in a case-control comparison (37 late-onset sepsis/NEC infants vs. 37 nonsepsis infants). In order to prevent any systemic bias, a stringent study design involving analysis of longitudinal plasma samples (10 sepsis/NEC infants vs. 10 nonsepsis infants) was included to ensure the pathophysiologic relevance of these proteins (Figure 1). Moreover, to integrate the diagnostic values of the potential biomarkers and to develop an economical and clinically practical assay, biomarkers with relevant but nonredundant diagnostic values were selected by multivariate logistic regression for construction of a diagnostic equation. The potential biomarkers and the diagnostic equation were then validated with an independent set of case-control cases (40 late-onset sepsis/NEC infants vs. 40 nonsepsis infants). The diagnostic equation was further subjected to validation in a prospective cohort study ( $n=104$ sus- pected sepsis/NEC episodes) to determine its diagnostic utilities, including positive and negative predictive values, for diagnosing late-onset septicemia and/or NEC and to formulate a new antibiotic treatment strategy for preterm infants.

\section{Results}

Clinical characteristics of studied infants. There were no significant differences in clinical characteristics between late-onset sepsis/NEC and nonsepsis infants, including gestational age, birth weight, Apgar scores, postnatal age of sepsis screening, and use of systemic corticosteroids after birth (Table 1). As expected, parameters that were associated with disease severity such as C-reactive protein (CRP) levels, incidence of DIC, requirement for vasopressor support, and death, were significantly higher in the sepsis/NEC group (Tables 1 and 2). 


\section{Table 1}

Demographic and clinical characteristics of 77 sepsis/NEC (group 1) and 77 nonsepsis infants (group 2) in the case-control study

\begin{tabular}{|c|c|c|c|c|c|c|}
\hline \multirow[t]{2}{*}{ Clinical features } & \multicolumn{6}{|c|}{ Biomarker discovery setIndependent validation set } \\
\hline & $\begin{array}{l}\text { Nonsepsis } \\
(n=37)\end{array}$ & $\begin{array}{l}\text { Sepsis/NEC } \\
\quad(n=37)\end{array}$ & $P$ value & $\begin{array}{l}\text { Nonsepsis } \\
(n=40)\end{array}$ & $\begin{array}{l}\text { Sepsis/NEC } \\
\quad(n=40)\end{array}$ & $P$ value \\
\hline Gestational age (wk) & $26.7(25.1-29.6)$ & $28.4(27.7-29.6)$ & $0.016^{A}$ & $28.3(26.1-29.6)$ & $28.2(27.0-30.4)$ & 0.292 \\
\hline Birth weight $(\mathrm{g})$ & $924(784-1365)$ & $1070(879-1303)$ & 0.277 & $925(790-1265)$ & $1050(843-1370)$ & 0.189 \\
\hline Sex: male/female $(n)$ & $27(73 \%) / 10(27 \%)$ & $19(51 \%) / 18(49 \%)$ & 0.093 & $24(60 \%) / 16(40 \%)$ & $21(52.5 \%) / 19(47.5 \%)$ & 0.652 \\
\hline Inborn/outborn $(n)$ & $32(86 \%) / 5(14 \%)$ & $27(73 \%) / 10(27 \%)$ & 0.247 & $36(90 \%) / 4(10 \%)$ & $30(75 \%) / 10(25 \%)$ & 0.141 \\
\hline Apgar score, 1 minute & $7(6-9)$ & $7(6-8)$ & 0.873 & $7(5-9)$ & $8(6-8)$ & 0.275 \\
\hline Apgar score, 5 minutes & $8(8-9)$ & $9(8-9)$ & 0.515 & $9(8-10)$ & $9(8-9)$ & 0.544 \\
\hline $\begin{array}{l}\text { Postnatal age of sepsis/ } \\
\text { NEC evaluation (d) }\end{array}$ & $22.0(13.8-36.0)$ & $24.0(15.0-32.5)$ & 0.893 & $22.0(14.0-40.0)$ & $23.5(14.0-43.0)$ & 0.773 \\
\hline $\begin{array}{l}\text { Postnatal systemic } \\
\text { corticosteroids } \\
\text { treatment }(n)\end{array}$ & $11(30 \%)$ & $2(5 \%)$ & 0.015 & $8(20 \%)$ & $9(23 \%)$ & 1.000 \\
\hline \multicolumn{7}{|l|}{ Mechanical ventilation $(n)$} \\
\hline IPPV or HFOV & $10(27 \%)$ & $23(62 \%)$ & 0.009 & $12(30 \%)$ & $16(40 \%)$ & 0.084 \\
\hline nCPAP & $11(30 \%)$ & $7(19 \%)$ & & $16(40 \%)$ & $7(17.5 \%)$ & \\
\hline Nil & $16(43 \%)$ & $7(19 \%)$ & & $12(30 \%)$ & $17(42.5 \%)$ & \\
\hline Vasopressor support (n) & $0(0 \%)$ & $9(24 \%)$ & $0.001^{\mathrm{B}}$ & $0(0 \%)$ & $11(28 \%)$ & $<0.001^{\mathrm{B}}$ \\
\hline $\mathrm{CRP}(\mathrm{mg} / \mathrm{l})$ & $3.0(1.0-3.0)$ & $40(12.7-67.9)$ & $<0.001^{B}$ & $3.0(3.0-3.0)$ & $30.4(6.0-74.0)$ & $<0.001^{\mathrm{B}}$ \\
\hline $\operatorname{DIC}(n)$ & $1(3 \%)$ & $18(48 \%)$ & $<0.001^{B}$ & $0(0 \%)$ & $17(43 \%)$ & $<0.001^{\mathrm{B}}$ \\
\hline Platelet count $\left(\times 10^{9} / l\right)$ & $378(273-425)$ & $151(105-241)$ & $<0.001^{B}$ & $304(188-444)$ & $196(101-262)$ & $<0.001^{\mathrm{B}}$ \\
\hline \multicolumn{7}{|c|}{$\begin{array}{l}\text { Of } 77 \text { nonsepsis infants, } 36 \text { had acute exacerbation of bronchopulmonary dysplasia, } 16 \text { had gastrointestinal dysmotility, } 12 \text { had apnea of prematurity, } 5 \\
\text { had unstable temperature due to environmental factors, } 5 \text { had heart failure with ventricular septal defect or patent ductus arteriosus, } 2 \text { had severe anemia } \\
\text { and } 1 \text { had extravasation of parental nutrition fluid from the percutaneous long line. Results are number (\%) or median (interquartile range). } \chi^{2} \text { (with Yates } \\
\text { correction) was used for categorical variables and Mann-Whitney } U \text { test for numerical variables. Of } 77 \text { sepsis/NEC infants, } 37 \text { had proteomic profiling, } \\
\text { and } 40 \text { participated in the independent validation phase. Similarly, the same number of nonsepsis infants underwent the same } 2 \text { phases of investigation. } \\
\text { Of } 77 \text { sepsis/NEC infants, } 56 \text { had culture-proven septicemia and } 21 \text { had NEC. In NEC infants, } 7 \text { also had septicemia. The number of NEC patients was } \\
\text { evenly distributed in the proteomic profiling phase }(n=10) \text { and independent validation phase of investigation }(n=11) \text {. IPPV, intermittent positive pressure } \\
\text { ventilation; HFOV, high-frequency oscillatory ventilation; nCPAP, nasal continuous positive airway pressure. }{ }^{A T h e} \text { comparison of gestational ages in the } \\
\text { biomarker discovery set was not statistically significant after Bonferroni's correction. }{ }^{B} P<0.004 \text {, Bonferroni-adjusted } P \text { value cutoff for } 13 \text { comparisons. Nil } \\
\text { indicates no mechanical ventilation. }\end{array}$} \\
\hline
\end{tabular}

Identification of plasma proteomic peaks associated with sepsis and NEC. In the biomarker discovery data set, proteomic peaks $(m / z 2,000-250,000)$ across the mass spectra of plasma samples from 37 late-onset septic/NEC infants and 37 nonsepsis infants (i.e., controls) were matched, and normalized peak intensities of 180 proteomic peaks were obtained (Supplemental Figure 1; supplemental material available online with this article; doi:10.1172/JCI40196DS1). We identified 25 peaks that were significantly higher and 40 peaks that were significantly lower in the sepsis/NEC group.

To avoid proteomic peaks caused by systematic biases unrelated to sepsis/NEC, differential proteomic peaks identified in the discovery set were considered as potential biomarkers only if their levels demonstrated a reversal pattern upon disease recovery. Plasma samples were obtained in a longitudinal manner on days $0,1,3-5$, and 6-20 in 10 randomly selected nonsepsis cases, and on days $0,1,3-5$, and the day of complete recovery (also between day 6 and 20 after the onset) in 10 randomly selected sepsis/NEC cases. Spearman's rank-order correlation analysis showed that 10 of 65 (15\%) proteomic peaks had significant reversal of their peak intensities upon recovery in the sepsis/ NEC group, but no such reversal pattern was found in the nonsepsis group (Table 3 and Figure 2; Supplemental Figures 1, 2, and 3). In the sepsis/NEC group, proteomic peaks at $m / z 6,940$, $m / z 8,917, m / z 13,878, m / z 73,065$, and $m / z 146,873$ showed an increasing trend in peak intensity upon recovery, while those at $m / z 10,181, m / z 11,528, m / z 11,674, m / z 11,731$, and $m / z 11,918$ showed a decreasing trend upon recovery. These 10 proteomic peaks were selected as potential diagnostic markers. There were significantly more upregulated peaks and fewer downregulated peaks observed in the sepsis/NEC group on day 0 (Supplemental Table 2 ). The mean $m / z$ values (minimum-maximum), peak intensities, and statistical results of these 10 diagnostic peaks are summarized in Table 3.

Protein identities of diagnostic proteomic peaks. Mass spectrometric analyses of selected proteomes recovered from 2D gels showed that 4 of the diagnostic proteomic peaks were serum amyloid $\mathrm{A}$ (SAA) $(m / z 11,674)$, a des-arginine variant of SAA $(m / z 11,528)$, transthyretin $(m / z 13,878)$, and $\alpha$-1B-glycoprotein $(m / z 73,065)$ (Supplemental Table 3). Immunodepletion experiments showed that 3 of the diagnostic proteomic peaks were proapolipoprotein CII (Pro-apoC2) $(m / z 8,917), \beta_{2}$-microglobulin (B2MG) $(m / z, 11,731)$, and a variant of B2MG $(m / z 11,918)$ (Supplemental Figure 4). We were unable to obtain the protein identity of the peak at $m / z 10,181$. For the proteomic peaks at $m / z 6,940$ and $m / z 73,065$, no corresponding protein spots in the $2 \mathrm{D}$ gels were observed. However, the high correlation $(r=0.81, P<0.001)$ between the normalized peak intensities of proteomic peaks at $m / z 6,940$ and $m / z 13,878$ suggested that the proteomic peak at $m / z 6,940$ was contributed by the double-charged transthyretin ions. For the proteomic peak at $m / z 146,873$, the significant correlation $(r=0.70, P<0.001)$ between the normalized peak intensities of the proteomic peaks at $m / z 146,873$ and $m / z 73,065$ suggested 


\section{Table 2}

Demographic and clinical characteristics of 104 suspected sepsis/NEC episodes that occurred in 60 preterm infants in the prospective cohort validation study

Clinical features
Gestational age (wk)
Birth weight $(\mathrm{g})$
Sex: male/female $(n)$
Inborn/outborn $(n)$
Apgar score, 1 minute
Apgar score, 5 minutes
Postnatal age of sepsis/NEC evaluation $(\mathrm{d})$
Postnatal systemic corticosteroids treatment $(n)$
Mechanical ventilation $(n)$
$\quad$ IPPV or HFOV
$\quad$ nCPAP
$\quad$ Nil
Vasopressor support $(n)$
CRP $($ mg/l)
DIC $(n)$
Platelet count $(\times 10 \% / l)$

49 Nonsepsis
episodes
in 31 infants
$28.0(25.7-29.7)$
$870(740-1255)$
$37(76 \%) / 12(24 \%)$
$43(88 \%) / 6(12 \%)$
$6(5-7)$
$8(7-8)$
$33.0(19.0-79.0)$
$4(8 \%)$
$6(12 \%)$
$25(51 \%)$
$18(37 \%)$
$1(2 \%)$
$0.5(0.5-2.6)$
$0(0 \%)$
$317(229-453)$

42 Sepsis/
NEC episodes
in 30 infants
$31.0(27.3-34.4)$
$1320(915-2190)$
$24(57 \%) / 18(43 \%)$
$33(79 \%) / 9(21 \%)$
$7(6-8)$
$8(7-10)$
$41.5(19.0-60.0)$
$0(0 \%)$
$15(51 \%)$
$8(18 \%)$
$19(31 \%)$
$3(7 \%)$
$25.2(9.6-45.9)$
$19(45 \%)$
$216(111-328)$

13 Probable clinical

sepsis episodes

in 12 infants

$30.0(27.4-33.4)$

$1250(915-1615)$

$6(46 \%) / 7(54 \%)$

$10(77 \%) / 3(23 \%)$

$7(6-7)$

$8(7-10)$

$42.0(24.0-67.0)$

$0(0 \%)$

$3(23 \%)$

$3(23 \%)$

$7(54 \%)$

$0(0 \%)$

$28.0(13.7-36.2)$

$3(23 \%)$

$202(150-285)$

Of 49 nonsepsis episodes, 19 were acute exacerbation of bronchopulmonary dysplasia, 9 were gastrointestinal dysmotility, 9 were apnea of prematurity, 5 were unstable temperature due to environmental factors, and 2 were indomethacin-induced gastritis. There was also 1 case of each of the following: cow's milk protein allergy, spectum plugging with lung collapse, renal tubular acidosis, late presentation of congenital diaphragmatic hernia, and transient adrenal insufficiency of prematurity. All the descriptive statistics were based on episodes. Results are number (\%) or median (interquartile range).

that the $m / z 146,873$ peak was contributed by the single-charged dimer ions of $\alpha$-1B-glycoprotein. The protein identities of the diagnostic proteomic peaks are summarized in Table 4.

Plasma concentrations of transthyretin, SAA, B2MG, and apolipoprotein CII (apoC2) measured by immunoassay were demonstrated to be highly correlated with the normalized peak intensities of their corresponding proteomic peaks assessed by the magnetic bead-based proteomic profiling (Table 5). The plasma levels of transthyretin $(P<0.001)$ and apoC2 $(P<0.001)$ were significantly lower in the sepsis/NEC group, whereas levels of SAA $(P<0.001)$ and B2MG $(P=0.039)$ were significantly higher in the sepsis/NEC group. The areas under the receiver operating characteristic (ROC) curves of these proteins ranged from 0.61 to 0.92 (Table 5).

Construction of a diagnostic equation for identification of sepsis and $N E C$. In order to integrate the diagnostic values of the potential biomarkers and to develop an economical and clinically practical assay, multivariate logistic regression analyses were performed to select biomarkers with relevant but nonredundant diagnostic values for constructing a diagnostic equation. Based on the biomarker discovery cases $(n=37$ for each of sepsis/NEC and nonsepsis groups), multivariate logistic regression analysis of immunoassay concentrations of transthyretin, SAA, B2MG, and apoC2 identified plasma apoC2 $(P=0.011)$ and SAA $(P=0.007)$ as the only 2 nonredundant biomarkers. Thus, immunoassay concentrations of plasma apoC2 and SAA were used to construct an equation (see Methods) for calculating the "ApoSAA score," which was the diagnostic score used for identification of sepsis and/or NEC cases. Hosmer-Lemeshow statistic showed that there was no significant evidence of lack of fit. In the equation, the negative coefficient $(-0.067)$ of apoC2 and positive coefficient $(0.1635)$ of SAA were consistent with the observations that apoC2 and SAA were negatively and positively associated with the outcome, respectively.
Independent validation of biomarkers and the ApoSAA score. Diagnostic values of the proteomic peaks, immunoassay concentrations of identified plasma proteins (transthyretin, apoC2, SAA, B2MG), and the ApoSAA score were further assessed in an independent validation case-control cohort of 40 late-onset sepsis/NEC and 40 nonsepsis infants. In agreement with the discovery set, the normalized peak intensities of proteomic peaks at $m / z 6,940$, $m / z 8,917, m / z 13,878, m / z 73,065$, and $m / z 146,873$ (Table 3) as well as plasma concentrations of transthyretin and apoC2 (Table 5) were significantly lower in sepsis/NEC patients, whereas the normalized peak intensities of proteomic peaks at $m / z 10,181$, $m / z 11,528, m / z 11,674, m / z 11,731$, and $m / z 11,918$ (Table 3 ) as well as the plasma concentrations of SAA and B2MG (Table 5) were significantly higher in the sepsis/NEC group. ROC curve analyses revealed that the identified plasma proteins (all $P<0.010$ ) were potentially useful biomarkers, with areas under the ROC curves of these proteins ranging from 0.69 to 0.96 (Table 5).

Furthermore, the ApoSAA scores were significantly higher in sepsis/NEC patients $(0.901,95 \% \mathrm{CI}=0.826-0.976)$ than in nonsepsis infants $(0.220,95 \% \mathrm{CI}=0.156-0.284, P<0.001$; Table 5$)$. The area under the ROC curve of the ApoSAA score was 0.96 (95\% CI $=0.93-1.00, P<0.001$; Figure 3$)$, which was significantly higher $(P=0.011, z$ statistics) than that of CRP (area under the ROC curve $=0.86,95 \% \mathrm{CI}=0.76-0.97, P<0.001)$. The diagnostic utilities of the ApoSAA score (i.e., sensitivity and specificity) at various cutoff values are summarized in Table 6 . In essence, at the cutoff value of 0.750 , the sensitivity and specificity were $90 \%$ and $95 \%$, respectively. At 0.199 , the specificity was $68 \%$, while both sepsis cases and NEC cases were identified at very high sensitivity of $100 \%$ and $83 \%$, respectively. At 0.170 , the sensitivity and specificity were $100 \%$ and $55 \%$, respectively. In contrast, when apoC2, SAA, and CRP were individually assessed at the maximum sensitivity 


\section{Table 3}

Intensity of preterm neonatal sepsis/NEC-associated proteomic peaks identified by mass spectrometry-based plasma proteomic profiling in the biomarker discovery and independent case-control validation sets

\begin{tabular}{|c|c|c|c|c|c|c|c|}
\hline \multirow{3}{*}{$\begin{array}{l}\text { Proteomic peaks }{ }^{A} \\
\text { Mean } m / z \text { values } \\
\text { (range) }\end{array}$} & \multicolumn{4}{|c|}{ Biomarker discovery set } & \multicolumn{3}{|c|}{ Independent validation set } \\
\hline & \multirow{2}{*}{$\begin{array}{l}\text { Correlation with days } \\
\text { of follow-up in the } \\
\text { sepsis/NEC Group, } \\
r \\
(P \text { values })\end{array}$} & \multicolumn{3}{|c|}{$\begin{array}{c}\text { Normalized peak intensity }(\%) \text {, } \\
\text { mean } \pm \text { SD }\end{array}$} & \multicolumn{3}{|c|}{$\begin{array}{c}\text { Normalized peak intensity (\%), } \\
\text { mean } \pm \text { SD }\end{array}$} \\
\hline & & $\begin{array}{l}\text { Nonsepsis } \\
\text { infants } \\
(n=37)\end{array}$ & $\begin{array}{c}\text { Sepsis/NEC } \\
\text { infants } \\
(n=37)\end{array}$ & $\begin{array}{c}\text { Student's } \\
t \text { test } \\
\text { (P values) }\end{array}$ & $\begin{array}{l}\text { Nonsepsis } \\
\text { infants } \\
(n=40)\end{array}$ & $\begin{array}{l}\text { Sepsis/NEC } \\
\text { infants } \\
(n=40)\end{array}$ & $\begin{array}{c}\text { Student's } \\
\text { t test } \\
\text { (P values) }\end{array}$ \\
\hline 6940 (6933-6945) & $0.24(0.048)$ & $0.24 \pm 0.22$ & $0.11 \pm 0.10$ & $\left(0.001^{\mathrm{B}}\right)$ & $0.24 \pm 0.19$ & $0.09 \pm 0.05$ & $\left(<0.001^{B}\right)$ \\
\hline 8917 (8916-8920) & $0.31(0.010)$ & $1.71 \pm 0.77$ & $0.59 \pm 0.55$ & $\left(<0.001^{\mathrm{B}}\right)$ & $1.55 \pm 0.67$ & $0.67 \pm 0.44$ & $\left(<0.001^{\mathrm{B}}\right)$ \\
\hline $10181(10178-10183)$ & $-0.24(0.050)$ & $0.12 \pm 0.06$ & $0.19 \pm 0.09$ & $\left(<0.001^{B}\right)$ & $0.12 \pm 0.06$ & $0.18 \pm 0.08$ & $\left(<0.001^{B}\right)$ \\
\hline $11528(11526-11531)$ & $-0.31(0.010)$ & $0.02 \pm 0.011$ & $0.28 \pm 0.51$ & $\left(0.005^{B}\right)$ & $0.02 \pm 0.01$ & $0.31 \pm 0.32$ & $\left(<0.001^{B}\right)$ \\
\hline $11674(11655-11687)$ & $-0.30(0.013)$ & $0.07 \pm 0.03$ & $0.68 \pm 1.05$ & $\left(0.001^{\mathrm{B}}\right)$ & $0.08 \pm 0.05$ & $0.66 \pm 0.71$ & $\left(<0.001^{\mathrm{B}}\right)$ \\
\hline 11731 (11724-11743) & $-0.32(0.008)$ & $0.24 \pm 0.09$ & $0.40 \pm 0.20$ & $\left(<0.001^{\mathrm{B}}\right)$ & $0.22 \pm 0.09$ & $0.47 \pm 0.21$ & $\left(<0.001^{B}\right)$ \\
\hline 11918 (11901-11918) & $-0.26(0.034)$ & $0.05 \pm 0.02$ & $0.12 \pm 0.11$ & $\left(<0.001^{B}\right)$ & $0.05 \pm 0.02$ & $0.12 \pm 0.08$ & $\left(<0.001^{B}\right)$ \\
\hline $13878(13846-13886)$ & $0.30(0.014)$ & $0.48 \pm 0.27$ & $0.19 \pm 0.15$ & $\left(<0.001^{\mathrm{B}}\right)$ & $0.45 \pm 0.25$ & $0.22 \pm 0.13$ & $\left(<0.001^{B}\right)$ \\
\hline 73065 (72941-73229) & $0.26(0.031)$ & $1.41 \pm 0.18$ & $1.17 \pm 0.25$ & $\left(<0.001^{B}\right)$ & $1.44 \pm 0.18$ & $1.32 \pm 0.25$ & $\left(0.002^{B}\right)$ \\
\hline $146873(146641-147163)$ & $0.26(0.032)$ & $0.60 \pm 0.12$ & $0.49 \pm 0.09$ & $\left(<0.001^{B}\right)$ & $0.63 \pm 0.14$ & $0.54 \pm 0.15$ & $(0.008)^{\prime}$ \\
\hline
\end{tabular}

${ }^{A} A l l$ differential proteomic peaks were identified at a false discovery rate of zero. ${ }^{B} P<0.005$ (Bonferroni-adjusted $P$ value cutoff for 10 comparisons).

of $100 \%$, their specificities were significantly lower, being $17.5 \%$, $2.5 \%$, and $0 \%$, respectively $(P=0.046, P<0.001$ and $P<0.001$; McNemar's test).

A prospective validation study on the diagnostic performance of the ApoSAA score. The diagnostic value of the ApoSAA score was further validated in a prospective cohort study. Of 104 suspected sepsis/NEC episodes, 31 were proven sepsis with positive blood culture, 11 were NEC ( $\geq$ grade II), 13 were probable clinical sepsis, and 49 were nonsepsis episodes (Table 2). For 100 of the 104 episodes, plasma samples were also collected 24 hours later (day 1 ).

At the time of clinical presentation (day 0), plasma levels of apoC2 were significantly lower in the proven sepsis/NEC group $(29.9 \mu \mathrm{g} / \mathrm{ml}, 95 \% \mathrm{CI}=22.6-37.2)$ than the nonsepsis group $(49.7 \mu \mathrm{g} / \mathrm{ml}, 95 \% \mathrm{CI}=37.2-62.3)$, whereas the plasma levels of SAA (sepsis $/ \mathrm{NEC}=197.1 \mu \mathrm{g} / \mathrm{ml}, 95 \% \mathrm{CI}=122.3-271.9$; nonsepsis $=$ $32.7 \mu \mathrm{g} / \mathrm{ml}, 95 \% \mathrm{CI}=0.0-74.3)$ and ApoSAA scores were significantly higher (sepsis/NEC $=0.904,95 \% \mathrm{CI}=0.830-0.977$; nonsepsis $=0.273,95 \% \mathrm{CI}=0.179-0.367)$ (Supplemental Table 4). There were no significant differences in the apoC2 levels, SAA levels, and ApoSAA scores between the sepsis group and the NEC group as well as between the sepsis group and the probable clinical sepsis group. A similar pattern was also observed when day 1 samples were examined (Supplemental Table 4).

ROC analysis confirmed that the ApoSAA score on day 0 was useful in the diagnosis of probable clinical sepsis, sepsis, and NEC (area under the ROC curve $=0.93,95 \% \mathrm{CI}=0.87-0.98, P<0.001$; Figure 3). The optimal cutoff values were selected to achieve sensitivities greater than $90 \%$ for diagnosing septicemia and NEC. With the cutoff value of 0.199 on day 0 , high sensitivities were obtained for identification of cases with probable clinical sepsis, proven sepsis, and NEC (100\%, 97\%, and 91\%, respectively; Table 6), and the positive predictive value was $82 \%$. At a cutoff value of 0.099 , all cases of probable clinical sepsis, proven sepsis, and NEC were identified by the ApoSAA score, and the specificity was $45 \%$ (95\% $\mathrm{CI}=31 \%-59 \%$, which was significantly higher than the specific- ity of CRP $(0 \%, P<0.001$, McNemar's test) at $100 \%$ sensitivity. In the next step, we attempted to increase the diagnostic sensitivity by examining both day 0 and day 1 samples. The diagnosis was considered positive when the ApoSAA score on either day 0 or day 1 was higher than the diagnostic cutoff. Thus, at the cutoff value of 0.199 , a negative predictive value of $100 \%$ was achieved, and the positive predictive value was $75 \%(95 \% \mathrm{CI}=65 \%-85 \%$; Table 6$)$.

A triage strategy for suspected sepsis/NEC infants. The excellent diagnostic performance of the ApoSAA score allows us to propose a strategy to clinically triage suspected late-onset sepsis/NEC infants into 3 risk category groups by using 2 cutoff values, 0.199 and 0.099 (Figure 4). As infants with probable clinical sepsis were likely to be genuinely infected, these patients were included in the sepsis/NEC group for triage purposes. On day 0, suspected infants with ApoSAA scores grater than 0.199 would be classified as having a high risk of developing sepsis/NEC, infants with scores between 0.099 and 0.199 as medium risk, and those with scores less than 0.099 as low risk. Prompt antibiotic treatment should be prescribed for the high and medium-risk groups. Importantly, the negative predictive value of the low-risk group is $100 \%$ and implies that none of the proven sepsis (including probable clinical sepsis) and NEC infants would be mistakenly classified into the nonsepsis group. Thus, the low-risk cases could be confidently excluded from starting antibiotic therapy. For the medium-risk group, a second blood test should be performed 24 hours later (day 1). A second ApoSAA score greater than 0.199 indicates a very high likelihood of sepsis/NEC. Such cases should be reclassified as being high risk, and a full course of antimicrobial treatment should be given. However, if the second ApoSAA score is less than 0.199, mediumrisk patients would be reclassified as being low risk and antibiotics could be stopped. This triage strategy has provided a negative predictive value of $100 \%$, and none of the sepsis/NEC cases were deprived of their life-saving antibiotic treatment. The positive predictive value for sepsis/NEC in the high-risk group was $75 \%$ ( $95 \% \mathrm{CI}=65 \%-85 \%$ ), implying that only $25 \%$ were false-positive 

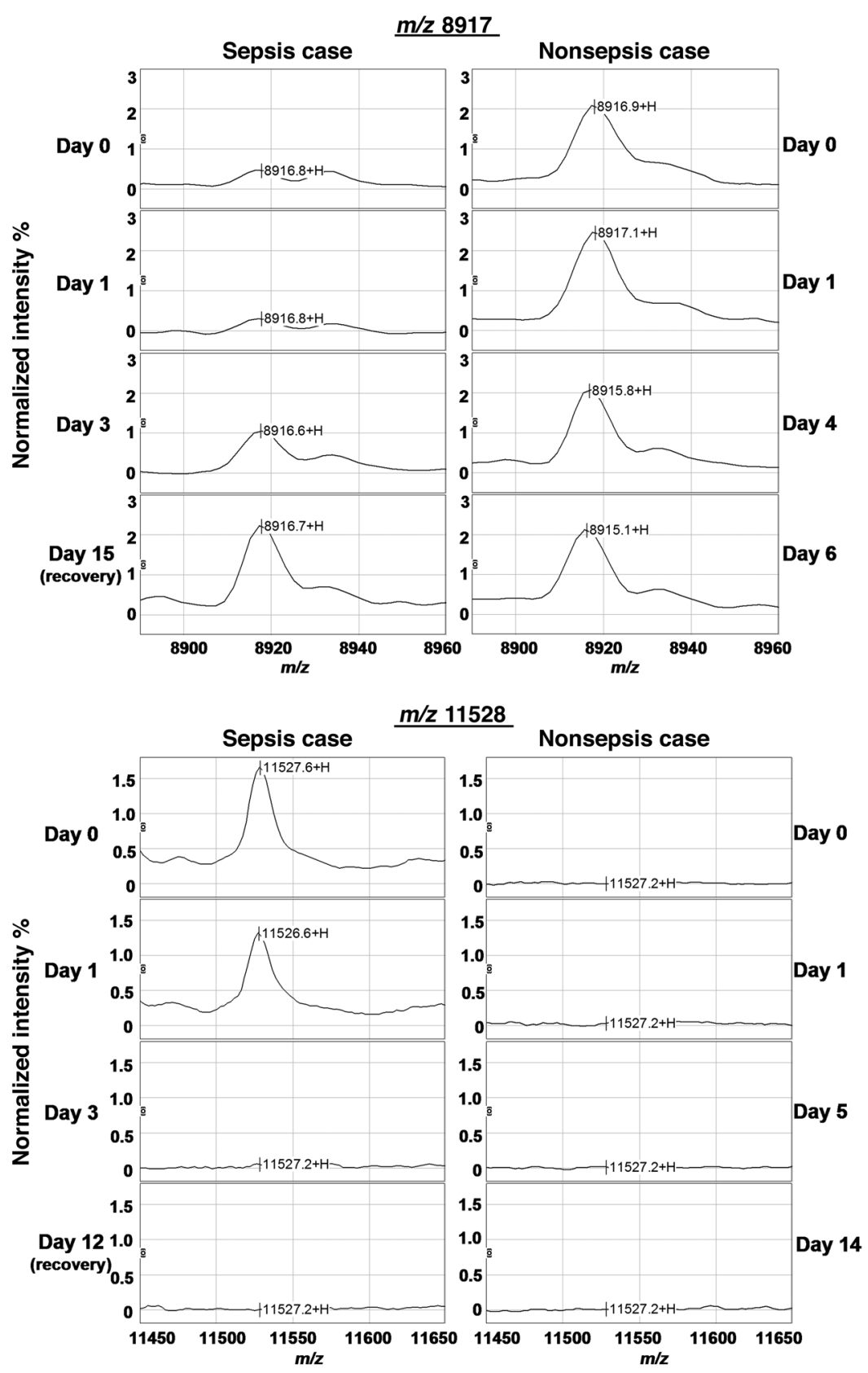

Figure 2

Representative mass spectra of the 2 biomarkers at $\mathrm{m} / \mathrm{z} 8,917$ and $\mathrm{m} / \mathrm{z} 11,528$ at the initial clinical presentation and during longitudinal follow-up.

cases. On day $0,45 \%(95 \% \mathrm{CI}=31 \%-59 \%)$ of nonsepsis cases could avoid starting unnecessary antibiotic treatment. On day 1 , an additional $16 \%$ of nonsepsis cases could be included in the low-risk group, resulting in a total of $61 \%(95 \% \mathrm{CI}=47 \%-75 \%)$ of nonsepsis cases either avoiding unnecessary antibiotic therapy or markedly shortening their antibiotic course to less than 24 hours.

Dysregulation of apolipoprotein metabolism in neonatal sepsis/NEC. By immunoassays, plasma concentrations of other apolipoproteins, including apoA1, apoA2, apoB, apoC3, and apoE on day 0 of all episodes involved in the biomarker discovery set, independent validation set, and prospective validation cohort (119 sepsis/NEC,
13 probable clinical sepsis and 126 nonsepsis cases) were measured. The results are summarized in Table 7. There was no significant difference in the apolipoprotein profiles among the probable clinical sepsis, sepsis, and NEC cases. The apolipoproteins could be classified into 3 categories. The first category consisted of SAA, the plasma concentration of which was significantly higher in the sepsis/NEC group. The second category consisted of apoA1, apoA2, apoC2, and apoC3, plasma concentrations of which were significantly decreased in sepsis/NEC episodes. The third category consisted of apoB and apoE, plasma concentrations of which were not significantly different between the sepsis/NEC and nonsepsis episodes.

\section{Discussion}

Early and accurate diagnosis of septicemia and NEC in preterm infants has always been a major challenge in neonatology. By undertaking the proteomic approach, we identified Pro-apoC2 $(\mathrm{m} / z$ 8917) and a des-arginine variant of SAA $(\mathrm{m} / z$ $11528)$ as useful biomarkers of neonatal septicemia and NEC. Based on the plasma concentrations of apoC2 and SAA evaluated by immunoassays, we were able to establish a simple and practical equation for calculating an ApoSAA score for early diagnosis of septicemia and NEC. The diagnostic value of the ApoSAA score was subsequently validated by both the independent case-control and prospective cohort studies. Importantly, the ApoSAA score has also provided a definitive strategy for safe and effective use of antimicrobial therapy for suspected preterm infants.

Biomarker discovery studies using mass spectrometry-based proteomic profiling technology are prone to systemic bias related to the nature of clinical samples, storage conditions, experimental design, spectrometric instruments, and bioinformatics analyses $(17,18)$. In the current study, a very stringent experimental design was adopted to minimize the chance of obtaining false-positive results. First, 3 separate groups of patients were used for diagnostic peak selection/equation training, independent case-control validation, and prospective cohort validation. Second, investigators were blinded to the clinical diagnoses while performing protein analysis and diagnostic equation development. Third, the false discovery rate of significant proteomic peaks was set at "zero." Fourth, differential proteomic peaks identified in the discovery set were considered as potential markers only if their levels demonstrated a reversal pattern upon disease recovery. The latter approach was to avoid proteomic peaks caused by systematic bias unrelated to sepsis/NEC. Among 65 differential proteomic peaks identified, only 10 (15\%) were selected as potential diagnostic markers because of this unique characteristic and no such pattern was encountered in the nonsepsis group. This crucial step ensured the pathophysiologic relevance of the biomarkers identified. More importantly, identities of most differential proteomic peaks were successfully characterized and 


\section{Table 4}

Protein identity of differential peaks, and correlations between the peak intensities and immunoassay concentrations

\begin{tabular}{|c|c|c|c|}
\hline $\begin{array}{l}\text { Diagnostic } \\
\text { proteomic } \\
\text { peaks, } m / z\end{array}$ & $\begin{array}{l}\text { Change in } \\
\text { sepsis/NEC }\end{array}$ & $\begin{array}{l}\text { Protein } \\
\text { identity }\end{array}$ & $\begin{array}{l}\text { Correlation } \\
\text { coefficient } \\
(P \text { values })^{A}\end{array}$ \\
\hline 6940 & Decrease & Transthyretin species & $0.547(<0.001)$ \\
\hline 8917 & Decrease & Pro-apoCII & $0.820(<0.001)$ \\
\hline 10181 & Increase & Unknown & NA \\
\hline 11528 & Increase & SAA variant (des-arginine) & $0.724(<0.001)$ \\
\hline 11674 & Increase & SAA & $0.693(<0.001)$ \\
\hline 11731 & Increase & $\mathrm{B} 2 \mathrm{MG}$ & $0.623(<0.001)$ \\
\hline 11918 & Increase & B2MG variant & $0.302(<0.001)$ \\
\hline 13878 & Decrease & Transthyretin & $0.635(<0.001)$ \\
\hline 73065 & Decrease & $\alpha-1 B-g l y c o p r o t e i n$ & NA \\
\hline 146873 & Decrease & $\alpha-1 B$-glycoprotein species & NA \\
\hline
\end{tabular}

${ }^{A}$ Correlation between normalized peak intensities and plasma levels of the identified proteins by immunoassay in 74 preterm infants ( 37 late-onset/NEC infants and 37 nonsepsis infants in the discovery phase) on day 0 ; all $P$ values $<0.007$, Bonferroniadjusted $P$ value cutoff for 7 comparisons. NA, not available.

similar results were obtained by immunoassay. Such findings confirmed that the proteomic peaks identified were not analytical artifacts. Fifth, the selected biomarkers were field tested and validated in a prospective cohort trial for determination of their diagnostic utilities. A limitation of the present proteomic protocol is that some proteins of low-plasma concentrations such as interleukins and chemokines may not be differentially detected. However, our stringent approach has identified a panel of vigorously regulated host response proteins of high plasma levels, and these candidate proteins should represent biomarkers with the best potential for "early" identification of neonatal septicemia and NEC.

Blood culture remains the "gold standard" in the diagnosis of neonatal septicemia. An adequate test typically requires at least $1 \mathrm{ml}$ of blood sample. It is well documented that obtaining sufficient quantity of blood from an ill neonate is difficult and therefore false negative results are common. In addition, despite the usefulness of the proteomic peaks for prediction of septicemia and NEC, their applications are not feasible in routine hospital laboratories lacking the proteomic facility. In contrast, quantifying plasma SAA and apoC2 proteins are straightforward and can be easily achieved by individual immunoassay or high throughput multiplex-assay platforms. The provision of absolute concentrations of these 2 biomarkers would allow intralaboratory quality control and interlaboratory comparison of results. Both SAA and apoC2 are present in plasma at high concentrations ( $\mu \mathrm{g}$ range) in neonates. Two microliters of plasma are adequate for measuring their circulating levels using commercial immunoassays. In addition, a fast turnaround time ( $<4$ hours) and high diagnostic accuracy can be readily achieved. Thus, we believe that the use of the ApoSAA score can be readily adopted in most current clinical settings.

The excellent diagnostic performance of the ApoSAA score allows us to develop what we believe to be a novel triage strategy to classify suspected late-onset septicemic and/or NEC infants into 3 distinct risk category groups (Figure 4). In the high-risk group, with ApoSAA score greater than 0.199 on day 0, prompt antimicrobial treatment and aggressive supportive care are mandatory. There would be minimal wastage of hospital resources due to inadvertent treatment of false-positive cases (Table 7). Sus- pected sepsis/NEC infants with ApoSAA score on day 0 between 0.099 and 0.199 should also be commenced on antibiotics, as this group contains both sepsis/ NEC and nonsepsis cases. However, antibiotics can be stopped early if the ApoSAA score 24 hours later (day 1 ) is less than 0.199. This is because ApoSAA scores of less than 0.199 on both consecutive days (day 0 and day 1) provide a $100 \%$ negative predictive value, at which none of the genuine sepsis/NEC infants would be wrongly classified as being nonsepsis cases. In the low-risk group, with ApoSAA score less than 0.099 at day 0 , antimicrobial treatment could be withheld, as none of the suspected infants developed sepsis/NEC in both our case-control and cohort validation studies. Thus, our overall findings provide a guideline for not starting antibiotic treatment at the time of clinical presentation if the ApoSAA score on day 0 is less than 0.099 and also for stopping unnecessary treatment after 24 hours in the medium-risk group when the day 1 ApoSAA score is less than 0.199. As far as we are aware, this is the first study using biomarkers to confidently exclude suspected infected/NEC infants from starting antimicrobial treatment. Thus, this triage strategy enables neonatologists to prescribe antibiotics rationally and to judiciously allocate resources by targeting those who truly require urgent treatment and intensive care. This strategy could preclude over half $(61 \%)$ of the true negative cases from receiving antibiotics unnecessarily or with very early stoppage of treatment. According to a multicenter survey in 2002 , it was suggested that more than $40 \%$ of VLBW infants received antibiotics needlessly (19). A recent study has also shown that prolonged antibiotic treatment could increase the risk of NEC (20). With our proposed strategy, a significant proportion of preterm infants can be precluded from unnecessary use of antimicrobial agents and therefore prevented from exposure to increased risk of NEC.

Of the 2 most promising proteins identified by proteomic profiling, SAA is a known marker of infection reported in newborns, whereas apoC2 has not been previously implicated. SAA is an acutephase reactant induced by proinflammatory cytokines (21) and can be stimulated directly by bacterial LPS. It is involved in cholesterol transport and can modulate immune responses, including cytokine production and activation (22), and chemotaxis of leukocytes (23). SAA has been reported to be a more effective biomarker than CRP or IL-6 in preterm infants because of its early, sensitive, and sustainable response to systemic infection (24). Thus, our current results are in accordance with recent clinical trials regarding its reliability for predicting septicemia and NEC in preterm infants (24-26).

Synthesized as Pro-apoC2 in the liver and gastrointestinal tract (27), apoC2 plays a crucial and complex role in plasma triglyceride metabolism and transport (28). It is a cofactor and an essential activator of lipoprotein lipase (29). Lower activities of lipoprotein lipase have been observed in preterm infants with sepsis, compared with those without sepsis (30). As far as we are aware, there has been no report describing a direct association between Pro-apoC2 or apoC2 and infection or colitis in patients of any age group. However, it has long been recognized that hyperlipidemia is a common phenomenon in preterm infants with severe infection. It is, therefore, plausible that downregulation of apoC2 during sepsis could lower lipoprotein lipase activity, leading to decreased hydrolysis and impaired clearance of triglyceride, 


\section{Table 5}

Immunoassay concentrations of the plasma proteins identified as potential diagnostic markers in the biomarker discovery and independent case-control validation sets

\begin{tabular}{|c|c|c|c|c|}
\hline \multirow[t]{2}{*}{ Identified proteins } & \multicolumn{3}{|c|}{ Plasma concentrations, mean $( \pm$ SD) } & \multirow{2}{*}{$\begin{array}{c}\text { ROC curve area } \\
(95 \% \mathrm{CI}) \\
(P \text { values })\end{array}$} \\
\hline & Nonsepsis & Sepsis/NEC & $\begin{array}{c}\text { Student's } t \text { test } \\
\text { ( } P \text { values) }\end{array}$ & \\
\hline \multicolumn{5}{|c|}{ Discovery group (37 nonsepsis cases, 37 sepsis/NEC cases) } \\
\hline $\begin{array}{l}\text { Transthyretin } \\
\text { apoC2 } \\
\text { SAA } \\
\text { B2MG }\end{array}$ & $\begin{array}{c}138.2( \pm 43.2) \\
42.9( \pm 21.2) \\
3.6( \pm 5.3) \\
4.6( \pm 1.4)\end{array}$ & $\begin{array}{c}103.9( \pm 42.2) \\
19.8( \pm 15.0) \\
55.4( \pm 39.7) \\
5.7( \pm 2.8)\end{array}$ & $\begin{aligned}<0.001^{A} \\
<0.001^{A} \\
<0.001^{A} \\
0.039\end{aligned}$ & $\begin{array}{c}0.73(0.62-0.85)\left(0.001^{A}\right) \\
0.86(0.77-0.94)\left(<0.001^{A}\right) \\
0.92(0.85-0.99)\left(<0.001^{A}\right) \\
0.61(0.48-0.74)(0.110)\end{array}$ \\
\hline \multicolumn{5}{|c|}{ Validation group (40 nonsepsis cases, 40 sepsis/NEC cases) } \\
\hline $\begin{array}{l}\text { Transthyretin } \\
\text { apoC2 } \\
\text { SAA } \\
\text { B2MG } \\
\text { ApoSAA score }\end{array}$ & $\begin{array}{c}136.5( \pm 53.3) \\
31.3( \pm 13.1) \\
4.1( \pm 6.5) \\
3.9( \pm 1.2) \\
0.220( \pm 0.204)\end{array}$ & $\begin{array}{c}105.0( \pm 35.9) \\
19.1( \pm 9.2) \\
56.5( \pm 34.9) \\
6.3( \pm 2.8) \\
0.901( \pm 0.239)\end{array}$ & $\begin{aligned} & 0.003^{B} \\
< & 0.001^{B} \\
< & 0.001^{B} \\
< & 0.001^{B} \\
< & 0.001^{B}\end{aligned}$ & $\begin{array}{c}0.69(0.57-0.81)\left(0.006^{\mathrm{B}}\right) \\
0.79(0.69-0.89)\left(<0.001^{\mathrm{B}}\right) \\
0.94(0.87-1.01)\left(<0.001^{\mathrm{B}}\right) \\
0.81(0.72-0.91)\left(<0.001^{* *}\right) \\
0.96(0.93-1.00)\left(<0.001^{\mathrm{B}}\right)\end{array}$ \\
\hline
\end{tabular}

A $P<0.013$, Bonferroni-adjusted $P$ value cutoff for 4 comparisons in the discovery set; ${ }^{B} P<0.010$, Bonferroni-adjusted $P$ value cutoff for 5 comparisons in the validation set.

resulting in clinical hyperlipidemia. High circulating triglyceride and/or triglyceride-rich lipoprotein levels may be protective against infection by neutralizing LPS.

It is of significance that both biomarkers identified by our proteomic analysis are apolipoproteins, suggesting an intimate relationship between lipoprotein metabolism and sepsis/NEC in preterm infants. These findings prompted us to investigate the characteristics of other related apolipoproteins. To our knowledge, the current study is the first to provide new information on changes of apoA1, apoA2, apoB, apoC2, apoC3, and apoE in plasma of preterm infants at an early phase of infection and/or NEC. apoA1, apoA2, apoC2, and apoC3 were significantly downregulated in sepsis/NEC infants compared with nonsepsis infants. There was also a trend of lower levels of apoB in the former group. In contrast, the plasma concentrations of apoE did not differ significantly between the groups. It is well documented that all lipoprotein classes can bind to LPS, which will then trigger a cascade of inflammatory responses. In adult patients, it has also been shown that plasma levels of apoA1, apoA2, and apoB were decreased during sepsis (31-35). Our results provide insights into the interplay and roles of various apolipoproteins in regulating the inflammatory cascade in preterm infants, but the mechanisms are likely to be complex and the exact pathophysiology warrants further exploration.

As these biomarkers have only been tested on preterm infants with late-onset sepsis/NEC, further prospective cohort trials are required to confirm their usefulness in early-onset sepsis, which is mainly caused by group B Streptococcus infection and congenital pneumonia (36). In addition, common neonatal complications such as bronchopulmonary dysplasia and intraventricular hemorrhage could theoretically elicit an inflammatory response and might influence the circulating levels of SAA and apoC2. This effect was, however, not observed in the case-control and prospective cohort studies nor in our previous biomarker studies $(9,12,36-38)$. Despite the occurrence of these complications in both sepsis/NEC and nonsepsis infants, the 2 biomarkers were able to identify sepsis/NEC episodes with great accuracy. The localized and mild inflammatory response of these complications had not significant- ly affected the diagnostic utilities of SAA, apoC2, or the ApoSAA score. In the current trial, both the case-control and prospective cohort studies included very preterm infants of 23.9 and 24.0 weeks gestation, respectively. However, the effects of gestational age and postnatal age on circulating apoC2 and SAA levels have not been systematically evaluated. Thus, we propose that a multicenter clinical trial should be performed on a wide range of gestational and postnatal ages to further assess the algorithm of the ApoSAA score before its implementation for routine clinical practice.

In summary, we have identified 2 potentially very useful "early" warning biomarkers from a large consortium of proteins through a very stringent and robust protocol. The ApoSAA score, which is computed from plasma levels of SAA and apoC2, is a practical and clinically useful equation for early and accurate identification of preterm infants with septicemia and/or NEC. More importantly, the ApoSAA score can potentially help frontline neonatologists to formulate the most appropriate and rational antibiotic treatment strategy for different risk categories of suspected sepsis/ NEC patients. A multicenter clinical study should be performed to further evaluate the algorithm of the ApoSAA score in order to ensure that this score is safe and can be generalized to other neonatal units for the management of suspected neonatal sepsis and NEC. Our results also suggested new data on the vigorous and concerted response of specific apolipoproteins to sepsis and NEC, thus indicating the important interrelationship between lipid metabolism and the host defense. Future studies are warranted to further delineate the mechanism and the exact role of apolipoproteins in these potentially fatal conditions.

\section{Methods}

Ethics statement. The study was approved by the Clinical Research Ethics Committee of the Chinese University of Hong Kong. Written informed consent was obtained from parents for all study infants.

Study design. For practical clinical reasons, both septicemia and NEC were considered as closely related disease entities of infection/inflammation and they were studied together. This is because (a) both conditions occur frequently in preterm infants and it is important that they are iden- 

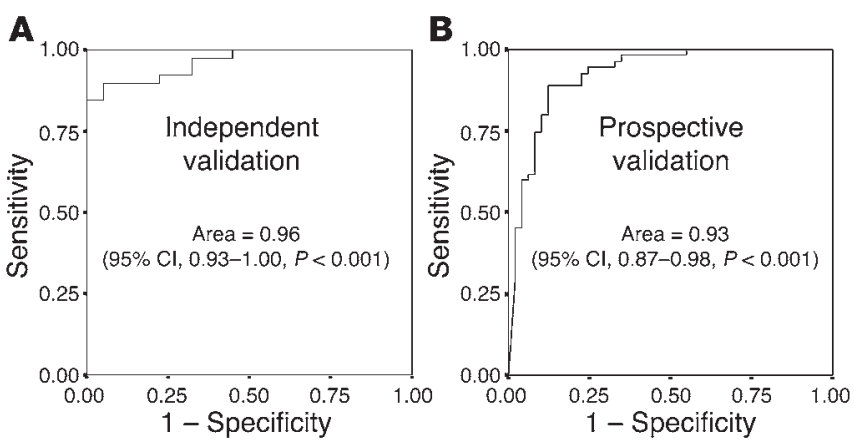

tified early; (b) their clinical presentations, which are nonspecific and subtle at the initial stage, are similar; (c) they may coexist in the same disease episode (i.e., NEC with septicemia occurred in one-third of cases of NEC in the case-control series); (d) our previous studies suggested that promising infection markers such as neutrophil CD64, IL-10, and RANTES were either significantly up- or downregulated in both conditions compared with nonsepsis cases $(12,37)$; and (e) most importantly, immediate medical management with prompt antimicrobial treatment and supportive care are identical for both conditions. Thus, it is of practical and clinical importance to identify these very similar but potentially lethal conditions at the earliest opportunity.

\section{Figure 3}

ROC curve analyses of day 0 ApoSAA score in detecting sepsis (including probable clinical sepsis) and NEC. Shown are the independent validation set (40 sepsis/NEC cases and 40 nonsepsis cases) (A) and prospective validation cohort (104 episodes of suspected clinical sepsis in 60 infants) (B).

Plasma samples of all cases were collected at the onset of clinical presentation during sepsis screening (day 0 ). In selected cases $(n=20)$, samples were obtained in a longitudinal and time sequential manner. The first part of the study was a case-control study that consisted of proteomic profiling and immunoassay analyses of candidate host-response biomarkers. This was followed by the second part of the study, using a prospective cohort of preterm infants to validate and to determine the diagnostic utilities of the newly discovered biomarkers.

In the first part of the study, the proteomic profiling process comprised 2 distinct phases: the biomarker discovery phase and the independent validation phase. In the biomarker discovery phase, a 3-stage approach was adopted, as follows: (a) the plasma proteomic profiles of 37 infants with proven sepsis/NEC (i.e., septicemia and/or NEC, group 1) were compared with those of 37 nonsepsis patients (group 2) to identify differential proteomic peaks. (b) These proteomic peaks were filtered upon the criterion of a reversal in expression pattern during longitudinal follow-up of 10 sepsis/NEC and 10 nonsepsis infants. (c)

\section{Table 6}

Summary of the diagnostic performance of ApoSAA score in the independent case-control validation set and prospective validation cohort at different cutoff values

Independent validation case-control set $\left(n=80^{\mathrm{A}}\right)$

Day 0 sample only

At cutoff value
Specificity ${ }^{\mathrm{B}} \%$ )
Sensitivity (\%)
Overall sensitivityC
Sepsis with positive blood
culture and/or NEC
Sepsis with positive blood
$\quad$ culture and with or
without NEC
NEC only
Probable clinical sepsis
Accuracy (\%)
Positive predictive value (\%)
Including probable
clinical sepsis cases
Excluding probable
clinical sepsis cases
Negative predictive value (\%)
Including probable
clinical sepsis cases
Excluding probable
clinical sepsis cases

$\begin{array}{cccc}\mathbf{0 . 7 5 0} & \mathbf{0 . 1 9 9} & \mathbf{0 . 1 7 0} & (\mathbf{n}) \\ 95 & 68 & 55 & (40) \\ & & & \\ 90 & 97 & 100 & (39) \\ 90 & 97 & 100 & (39) \\ 97 & 100 & 100 & (33)\end{array}$

$\begin{array}{llll}50 & 83 & 100 & (6) \\ \text { NA } & \text { NA } & \text { NA } & (0) \\ 92 & 82 & 77 & (79)\end{array}$

\begin{tabular}{|c|c|c|}
\hline NA & NA & NA \\
\hline S & NA & NA \\
\hline
\end{tabular}

Prospective validation cohort $(n=104)$

Day 0 sample only Prospective validation cohort
\[ (n=100) \]

Day 0 and day 1 samples, requiring 1 sample $>$ cutoff

$\begin{array}{cccccccc}\mathbf{0 . 7 5 0} & \mathbf{0 . 1 9 9} & \mathbf{0 . 1 7 0} & \mathbf{0 . 0 9 9} & \mathbf{( n )} & \mathbf{0 . 7 5 0} & \mathbf{0 . 1 9 9} & \mathbf{( n )} \\ 84 & 76 & 59 & 45 & (49) & 80 & 61 & (46) \\ & & & & & & & \\ 89 & 96 & 98 & 100 & (55) & 91 & 100 & (54) \\ 88 & 95 & 98 & 100 & (42) & 90 & 100 & (42) \\ 90 & 97 & 100 & 100 & (31) & 94 & 100 & (31)\end{array}$

$\begin{array}{cccccccc}\mathbf{0 . 7 5 0} & \mathbf{0 . 1 9 9} & \mathbf{0 . 1 7 0} & \mathbf{0 . 0 9 9} & (\boldsymbol{n}) & \mathbf{0 . 7 5 0} & \mathbf{0 . 1 9 9} & \mathbf{( n )} \\ 84 & 76 & 59 & 45 & (49) & 80 & 61 & (46) \\ & & & & & & & \\ 89 & 96 & 98 & 100 & (55) & 91 & 100 & (54) \\ 88 & 95 & 98 & 100 & (42) & 90 & 100 & (42) \\ 90 & 97 & 100 & 100 & (31) & 94 & 100 & (31)\end{array}$

$\begin{array}{ccccccc}82 & 91 & 91 & 100 & (11) & 82 & 100 \\ 92 & 100 & 100 & 100 & (13) & 92 & 100 \\ 87 & 87 & 80 & 74 & (104) & 86 & 82\end{array}$

86

$\begin{array}{lllll}86 & 82 & 73 & 67 & \text { NA } \\ 82 & 77 & 67 & 61 & \text { NA }\end{array}$

$84 \quad 75$

NA

$81 \quad 70$

NA

$\begin{array}{llllllllllll}\text { NA } & \text { NA } & \text { NA } & \text { NA } & 87 & 95 & 97 & 100 & \text { NA } & 88 & 100 & \text { NA } \\ \text { NA } & \text { NA } & \text { NA } & \text { NA } & 89 & 95 & 97 & 100 & \text { NA } & 90 & 100 & \text { NA }\end{array}$

6)

1)




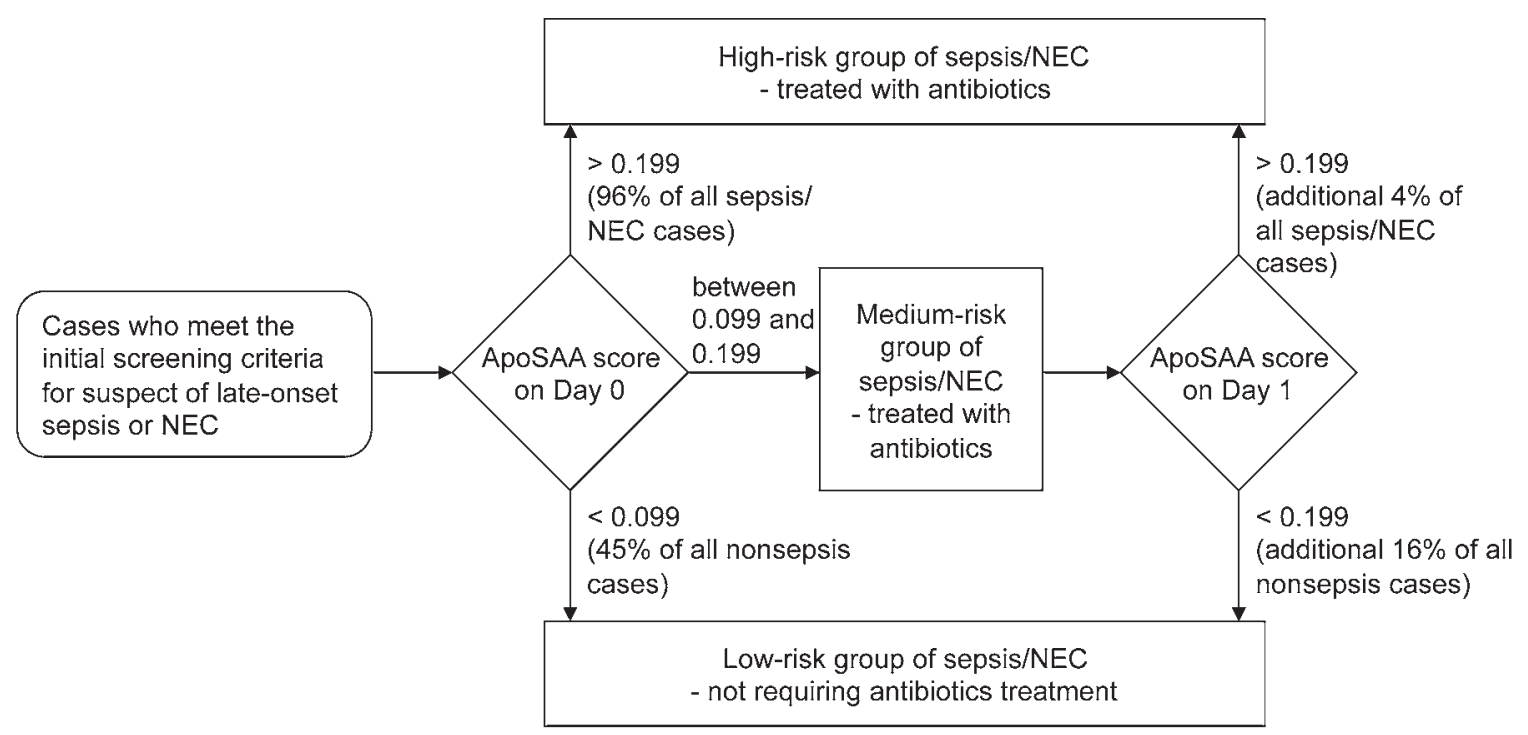

Figure 4

A triage strategy for classifying cases suspected for late-onset sepsis/NEC on the basis of ApoSAA scores on day 0 and day 1 .

The plasma concentrations of the identified proteins were subjected to logistic regression analysis for identifying the relevant nonredundant biomarkers. The plasma concentrations of these biomarkers were used to construct a diagnostic equation. In the subsequent independent validation phase, plasma samples (day 0) from 40 sepsis/NEC and 40 nonsepsis independent infants were evaluated by the diagnostic equations to determine their areas under the ROC curves.

In the second part of the study, the 2 candidates host-response biomarkers were subjected to further validation in a prospective cohort study in which "consecutive" suspected sepsis or NEC cases were evaluated. The optimal cutoff value and diagnostic utilities (sensitivity, specificity, positive, and negative predictive values) of these biomarkers and the computed diagnostic equation were determined. The study design and patient flow are summarized in Figure 1.

For clinical relevance, we included suspected sepsis/NEC cases with very similar signs and symptoms but caused by nonsepsis etiologies as controls. This strategy ensured that the clinical features per se did not elicit an inflammatory response that would influence the process of biomarker discovery. Asymptomatic preterm infants were not recruited as a separate control group for the following reasons: (a) those cases with longitudinal sampling could demonstrate both the acute symptomatic and asymptomatic (i.e., recovered phase) status of the patient; (b) in our previous studies, data on healthy controls did not significantly contribute to the process of biomarker evaluation $(37,38,39)$ and in subsequent trials, this group was not included $(9,12)$; and (c) for ethical reasons, we tried to minimize unnecessary blood taking in healthy preterm infants.

Patients. Preterm infants in the neonatal intensive care unit (NICU) at the Prince of Wales Hospital, Hong Kong, with (a) birth weight of less than 1,500 g; (b) postnatal age greater than 72 hours; (c) signs and symptoms suggestive of systemic infection or NEC and requiring full sepsis screening with intravenous antimicrobial coverage; and (d) written parental consent, were eligible for recruitment into the study. Infants were excluded if they were already receiving antibiotic treatment at the time of sepsis/NEC evaluation or had lethal congenital and chromosomal abnormalities $(37,38)$. In the case-control study, 2 distinct categories of cases were defined. Group 1, the proven sepsis/NEC group $(n=77)$, comprised infants with sepsis episodes that had been confirmed as bacterial or fungal culture-positive infection (i.e., septicemia with or without meningitis or peritonitis) or NEC cases (i.e., stage II or above in Bell's classification) (40). A list of commonly identified bacterial infections in our unit is described in Supplemental Table 1 (12). Group 2, the nonsepsis group $(n=77)$, consisted of infants who met the initial screening criteria for suspected clinical sepsis but were subsequently classified as being noninfected and had a definitive diagnosis unrelated to sepsis or NEC. Blood samples were collected from eligible infants at the initial clinical presentation (day 0) during sepsis/NEC screening. For longitudinal monitoring, blood samples were obtained on days $0,1,3-5$, and 6-20 in the nonsepsis group ( $n=10)$, and on days $0,1,3-5$, and the day of complete recovery (i.e., defined as the day immediately after cessation of antibiotic treatment, also between day 6 and 20 after the onset of presentation) in the sepsis/NEC group $(n=10)$. In the subsequent prospective cohort study, preterm infants with suspected infection and NEC ( $n=104$ episodes) were consecutively recruited. Blood samples were obtained on day 0 and day 1. Details of clinical features, sepsis/NEC screening, and classification criteria of sepsis/NEC and nonsepsis cases were described in detail in our previous reports $(12,37)$. In addition to groups 1 and 2, a probable clinical sepsis group (group 3 ) in which suspected infants presented with at least 3 clinical signs and symptoms plus evidence of hematologic or metabolic derangements despite negative bacterial culture were also considered to have sepsis based on strong circumstantial background of the clinical course and laboratory results. The analysis in the prospective cohort was based on episodes and not individual infants, thus simulating day-to-day clinical practice. The neonatologists who classified the consecutive episodes were blinded to the results of SAA and apoC2 (see Supplemental Methods). The recruitment of infection/NEC episodes was conducted between September 2002 and August 2007 in the case-control study and between January 2008 and September 2009 in the prospective cohort study.

Collection of specimens. Blood samples $(1 \mathrm{ml})$ were collected from indwelling arterial catheters or by venipuncture into a prechilled EDTA bottle. Plasma samples were separated by centrifugation (1,900 $g$ for 5 minutes) at $4^{\circ} \mathrm{C}$ and stored in $200 \mu \mathrm{l}$ aliquots at $-80^{\circ} \mathrm{C}$ until analysis. The schedule of blood sampling in longitudinal cases coincided exactly with the timing 
Table 7

Apolipoprotein profiles in sepsis/NEC and nonsepsis episodes on day 0

\begin{tabular}{|c|c|c|c|c|c|c|c|c|}
\hline \multirow[t]{2}{*}{ Patient group } & \multirow{2}{*}{$\begin{array}{l}\text { Number of } \\
\text { episodes }\end{array}$} & \multicolumn{6}{|c|}{ Plasma concentration on day 0, mean \pm SD $(\mu \mathrm{g} / \mathrm{ml})$} & \multirow[b]{2}{*}{ SAA } \\
\hline & & apoA1 & apoA2 & apoB & apoC2 & apoC3 & apoE & \\
\hline Sepsis'A/NEC & 119 & $695 \pm 238$ & $122.7 \pm 78.4$ & $104.8 \pm 109.2$ & $23.2 \pm 18.1$ & $40.3 \pm 29.0$ & $111.5 \pm 67.7$ & $105.8 \pm 163.4$ \\
\hline Sepsis onlyA & 87 & $685 \pm 220$ & $125.0 \pm 76.0$ & $106.2 \pm 99.3$ & $22.7 \pm 19.0$ & $39.9 \pm 30.6$ & $114.6 \pm 68.7$ & $113.1 \pm 175.4$ \\
\hline NEC only & 26 & $756 \pm 297$ & $124.7 \pm 89.5$ & $108.5 \pm 142.4$ & $26.8 \pm 15.5$ & $44.2 \pm 25.7$ & $113.2 \pm 64.7$ & $90.4 \pm 134.3$ \\
\hline $\begin{array}{l}\text { Probable clinical } \\
\text { sepsis }^{B}\end{array}$ & 13 & $755 \pm 298$ & $73.2 \pm 23.7$ & $48.1 \pm 24.6$ & $25.0 \pm 14.0$ & $43.8 \pm 19.5$ & $127.0 \pm 71.4$ & $95.9 \pm 143.7$ \\
\hline $\begin{array}{l}\text { Nonsepsis }{ }^{\circ} \\
\text { Kruskal-Wallis test } \\
\quad P \text { value }\end{array}$ & 126 & $\begin{array}{c}935 \pm 289 \\
<0.001\end{array}$ & $\begin{array}{c}164.3 \pm 82.9 \\
<0.001\end{array}$ & $\begin{array}{c}139.8 \pm 159.6 \\
0.059\end{array}$ & $\begin{array}{c}41.9 \pm 31.9 \\
<0.001\end{array}$ & $\begin{array}{c}88.2 \pm 90.8 \\
<0.001\end{array}$ & $\begin{array}{c}107.8 \pm 56.9 \\
0.697\end{array}$ & $\begin{array}{c}15.1 \pm 93.2 \\
<0.001\end{array}$ \\
\hline \multicolumn{9}{|c|}{ Tukey's post-hoc test ( $P$ value, 2 tails) } \\
\hline \multicolumn{2}{|c|}{ Sepsis/NEC vs. nonsepsis } & $<0.001$ & $<0.001$ & 0.107 & $<0.001$ & $<0.001$ & 0.891 & $<0.001$ \\
\hline \multicolumn{2}{|c|}{ Sepsis vs. nonsepsis } & $<0.001$ & 0.003 & 0.287 & $<0.001$ & $<0.001$ & 0.868 & $<0.001$ \\
\hline \multicolumn{2}{|c|}{ NEC vs. nonsepsis } & 0.012 & 0.098 & 0.707 & 0.037 & 0.015 & 0.978 & 0.047 \\
\hline \multicolumn{2}{|l|}{ Sepsis vs. NEC } & 0.643 & 1.000 & 1.000 & 0.897 & 0.992 & 1.000 & 0.873 \\
\hline \multicolumn{2}{|c|}{$\begin{array}{l}\text { Probable clinical sepsis } \\
\text { vs. nonsepsis }\end{array}$} & 0.056 & $<0.001$ & 0.052 & 0.064 & 0.060 & 0.548 & 0.094 \\
\hline \multicolumn{2}{|c|}{$\begin{array}{l}\text { Probable clinical sepsis } \\
\text { vs. sepsis }\end{array}$} & 0.818 & 0.128 & 0.473 & 0.991 & 0.997 & 0.910 & 0.973 \\
\hline
\end{tabular}

AProven sepsis with positive blood culture. ${ }^{B}$ Probable clinical sepsis in which suspected infants presented with at least 3 clinical signs and symptoms plus evidence of hematologic or metabolic derangements despite negative bacterial culture. CNonsepsis cases who were suspected clinical sepsis but were subsequently classified as being noninfected and had a definitive diagnosis unrelated to sepsis or NEC.

for serial blood counts and CRP measurements or routine monitoring of blood gases, liver, and bone profiles of preterm infants, so as to minimize the disturbance to these infants.

Quantitative proteomic profiling. The plasma proteome of each sample was enriched with hydrophobic C18 magnetic beads (Dynal; Invitrogen). Extracted proteins were loaded in duplicate onto a gold ProteinChip array (Ciphergen Biosystems Inc.) and read on a ProteinChip PBS II reader (Ciphergen Biosystems) operating at MALDI-TOF mass spectrometry mode (41). Quantitative proteomic profiles (i.e., mass spectra) with a molecular mass ranging from 1.2 to $250 \mathrm{kDa}$ were acquired, externally calibrated, and processed, as previously described $(16,42)$. After smoothing and baseline subtraction, the common peaks among the mass spectra were identified and quantified using the Biomarker Wizard software. Both the intra-assay and inter-assay coefficient of variations of normalized peak intensities of the majority of peaks were between $4 \%$ and $30 \%$. The proteins corresponding to the proteomic peaks of interest were purified by $2 \mathrm{D}$ gel electrophoresis, and the identities were characterized by mass spectrometry as previously described or identified by immunodepletion approach $(15,43)$. Details of methods for proteomic profiling and protein identification are summarized in Supplemental Methods.

Quantifying selected markers by immunoassay. Plasma transthyretin, B2MG, SAA, apoC2, apoA1, apoA2, apoB, apoC3, and apoE were measured using commercial kits, according to the manufacturers' instructions (see Supplemental Methods for details).

Construction of the diagnostic equation. Multivariate logistic regression with forward stepwise (SPSS Inc.) was used to select the relevant nonredundant biomarkers for the development of the diagnostic equation and the scoring system for differentiating sepsis/NEC cases from nonsepsis cases. The diagnostic equation was evaluated for goodness of fit with the HosmerLemeshow statistic. Plasma concentrations of apoC2 and SAA measured by immunoassay were used for calculating the ApoSAA score: ApoSAA score $=1 /\{1+\exp [-(0.077-0.0674 \times$ apoC $2+0.1635 \times$ SAA $)]\}$, where apoC2 and SAA are plasma concentrations in $\mu \mathrm{g} / \mathrm{ml}$ and the ApoSAA score is the probability of having septicemia and/or NEC.
Statistics. The Mann-Whitney $U$ test, Student's $t$ test, and $\chi^{2}$ test were used to evaluate the demographic data, clinical characteristics, and biomarker levels between sepsis/NEC and nonsepsis infants (SPSS v16; SPSS Inc.). Two-tailed $P$ values were calculated. Unadjusted $P$ values with asterisks indicate those that remained statistically significant after Bonferroni's adjustment. For comparison of more than 2 groups, the Kruskal-Wallis test was performed. This was followed by Tukey or Dunnett's T3 post-hoc test for between group comparisons. $P<0.05$ was considered to be statistically significant. In the case-control study, the Significance Analysis of Microarray (Stanford University, Stanford, California, USA) was applied to identify proteomic peaks that were significantly different between the 2 groups $(16,42)$. In this program, 2 classed but unpaired data sets were selected as the data type, and 5,000 permutations were performed. The false discovery rate of significant differential peaks was set at zero to avoid identification of false significant proteomic peaks caused by multiple comparisons. The Spearman rank-order correlation test was used to identify any reversal in peak intensities among the significant proteomic peaks upon recovery of sepsis/NEC in infants with longitudinal blood sample monitoring. In the independent validation study and prospective cohort study, the ROC curve analysis was used to determine diagnostic values of the biomarkers. The optimal cutoff values were selected to achieve sensitivities of more than $90 \%$ for diagnosing septicemia and NEC. The corresponding specificity and positive and negative predictive values were calculated with the standard formulae.

\section{Acknowledgments}

The case-control study of this project was supported by the Research Fund for the Control of Infectious Diseases (RFCID, CUHK-BS-015) from the Food and Health Bureau of the Hong Kong SAR Government and by the Li Ka Shing Foundation. The prospective cohort study of this project was supported by the research grant (RGC reference no. CUHK471908 and project code 2140608) awarded by the Research Grant Council of the Government of Hong Kong, SAR. The funding organizations have no role in study design, data collection and analysis, decision to publish, or preparation of the 
manuscript. The authors are grateful for the technical support from Melody Y.M. Wong on the protein identification work.

Received for publication June 16, 2009, and accepted in revised form May 12, 2010.

1. Bassler D, et al. Trial of Indomethacin Prophylaxis
in Preterms Investigators. Using a count of neona-
tal morbidities to predict poor outcome in extreme-
ly low birth weight infants: added role of neonatal
infection. Pediatrics. 2009;123(1):313-318.
2. Lin PW, Nasr TR, Stoll BJ. Necrotizing enterocoli-
tis: recent scientific advances in pathophysiology
and prevention. Semin Perinatol. 2008;32(2):70-82.
3. Stoll BJ, et al. Late-onset sepsis in very low birth
weight neonates: the experience of the NICHD
Neonatal Research Network. Pediatrics. 2002;110(2
pt 1):285-291. 4. Ng PC, et al. Combined use of alcohol hand rub and gloves reduces the incidence of late onset infection in very low birthweight infants. Arch Dis Child Fetal Neonatal Ed. 2004;89(4):F336-F340.

5. Cole CR, Hansen NI, Higgins RD, Ziegler TR, Stoll BJ, Eunice Kennedy Shriver NICHD Neonatal Research Network. Very low birth weight preterm infants with surgical short bowel syndrome: incidence, morbidity and mortality, and growth outcomes at 18 to 22 months. Pediatrics. 2008;122(3):e573-e582.

6. Kaufman SS, Gondolesi GE, Fishbein TM. Parenteral nutrition associated liver disease. Semin Neonatol. 2003;8(5):375-381.

7. Adams-Chapman I, Stoll BJ. Neonatal infection and long-term neurodevelopmental outcome in the preterm infant. Curr Opin Infect Dis. 2006;19(3):290-297.

8. Stoll BJ, et al. Neurodevelopmental and growth impairment among extremely low-birthweight infants with neonatal infection. JAMA. 2004;292(19):2357-2365

9. Ng PC, et al. IP-10 is an early diagnostic marker for identification of late-onset bacterial infection in preterm infants. Pediatr Res. 2007;61(1):93-98.

10. Ng PC, Lam HS. Diagnostic markers for neonatal sepsis. Curr Opin Pediatr. 2006;18(2):125-131.

11. Ng PC, et al. High-dose oral erythromycin decreased the incidence of parenteral nutrition-associated cholestasis in preterm infants. Gastroenterology. 2007;132(5):1726-1739.

12. Ng PC, et al. Early prediction of sepsis-induced disseminated intravascular coagulation with interleukin-10, interleukin-6, and RANTES in preterm infants. Clin Chem. 2006;52(6):1181-1189.

13. Ng PC. Diagnostic markers of infection in neonates. Arch Dis Child Fetal Neonatal Ed. 2004; 89(3):F229-F235.

14. Buhimschi CS, et al. Proteomic profiling of the amniotic fluid to detect inflammation, infection, and neonatal sepsis. PLoS Med. 2007;4(1):e18.

15. Rüetschi U, et al. Proteomic analysis using protein chips to detect biomarkers in cervical and amniotic
Address correspondence to: Terence Chuen Wai Poon, Li Ka Shing Institute of Health Sciences, Department of Medicine and Therapeutics, The Chinese University of Hong Kong, Prince of Wales Hospital, Shatin, N.T., Hong Kong. Phone: 852.2632.1205; Fax: 852.2648.8842; E-mail: tcwpoon@cuhk.edu.hk. fluid in women with intra-amniotic inflammation. J Proteome Res. 2005;4(6):2236-2242.

16. Pang RT, et al. Serum proteomic fingerprints of adult patients with severe acute respiratory syndrome. Clin Chem. 2006;52(3):421-429.

17. Poon TC. Opportunities and limitations of SELDITOF-MS in biomedical research: practical advices. Expert Rev Proteomics. 2007;4(1):51-65.

18. Diamandis EP. Analysis of serum proteomic patterns for early cancer diagnosis: drawing attenting to potential problems. J Natl Cancer Inst. 2004;96(5):353-356

19. Stoll BJ, et al. Late-onset sepsis in very low birth weight neonates: the experience of the NICHD Neonatal Research Network. Pediatrics. 2002; 110(2 pt 1):285-291.

20. Cotten CM, et al. Prolonged duration of initial empirical antibiotic treatment is associated with increased rates of necrotizing enterocolitis and death for extremely low birth weight infants. Pediatrics. 2009;123(1):58-66.

21. Maury CP. Comparative study of serum amyloid A protein and C-reactive protein in disease. Clin Sci. 1985;68(2):233-238.

22. He R, Sang H, Ye RD. Serum amyloid A induces IL-8 secretion through a G protein-coupled receptor, FPRL1/LX4AR. Blood. 2003;101(4):1572-1581.

23. Badolato R, et al. Serum amyloid A is a chemoattractant: induction of migration, adhesion, and tissue infiltration of monocytes and polymorphonuclear leukocytes. J Exp Med. 1994; 180(1):203-209.

24. Arnon S, Litmanovitz I, Regev RH, Bauer S, Shainkin-Kestenbaum R, Dolfin T. Serum amyloid A: an early and accurate marker of neonatal early-onset sepsis. J Perinatol. 2007;27(5):297-302.

25. Arnon S, et al. Serum amyloid A protein is a useful inflammatory marker during late-onset sepsis in preterm infants. Biol Neonate. 2005;87(2):105-110.

26. Cetinkaya M, Ozkan H, Koksal N, Celebi S, Hacimustafaoglu M. Comparison of serum amyloid A concentrations with those of C-reactive protein and procalcitonin in diagnosis and follow-up of neonatal sepsis in premature infants. $J$ Perinatol. 2009;29(3):225-231.

27. Sharpe CR, Sidoli A, Shelley CS, Lucero MA, Shoulders CC, Baralle FE. Human apolipoproteins AI, AII, CIII. cDNA sequences and mRNA abundance. Nucleic Acids Res. 1984;12(9):3917-3932.

28. Jong MC, Hofker MH, Havekes LM. Role of ApoCs in lipoprotein metabolism functional differences between ApoC1, apoC2, and ApoC3. Arterioscler Thromb Vasc Biol. 1999;19(3):472-484.

29. Goldberg IJ, Scheraldi CA, Yacoub LK, Saxena U, Bisgaier CL. Lipoprotein apoC-II activation of lipo- protein lipase. J Biol Chem. 1990;265(8):4266-4272. 30. Rovamo L, Nikkilä EA, Taskinen MR, Raivio KO. Postheparin plasma lipoprotein and hepatic lipases in preterm neonates. Pediatr Res. 1984;18(11):1104-1107.

31. Khovidhunkit W, et al. Effects of infection and inflammation on lipid and lipoprotein metabolism: mechanisms and consequences to the host. J Lipid Res. 2004;45(7):1169-1196.

32. Li L, Thompson PA, Kitchens RL. Infection induces a positive acute phase apolipoprotein $\mathrm{E}$ response from a negative acute phase gene: role of hepatic LDL receptors. J Lipid Res. 2008;49(8):1782-1793.

33. Alvarez C, Ramos A. Lipids, lipoproteins, and apoproteins in serum during infection. Clin Chem. 1986;32(1 pt 1):142-145.

34. Barlage $S$, et al. Apo E-containing high density lipoproteins and phospholipids transfer protein activity increase in patients with a systemic inflammatory response. J Lipid Res. 2001;42(2):281-290.

35. Van Leeuwen HJ, Heezius EC, Dallinga GM, van Strijp JA, Verhoef J, van Kessel KP. Lipoprotein metabolism in patients with severe sepsis. Crit Care Med. 2003;31(5):1359-1366

36. Ng PC, et al. Neutrophil CD64 is a sensitive diagnostic marker for early-onset neonatal infection. Pediatr Res. 2004;56(5):796-803.

37. Ng PC, Li K, Wong RP, Chui KM, Wong E, Fok TF. Neutrophil CD64 expression: a sensitive diagnostic marker for late-onset nosocomial infection in very low birthweight infants. Pediatr Res. 2002;51(3):296-303.

38. Ng PC, et al. Diagnosis of late onset neonatal sepsis with cytokines, adhesion molecule, and C-reactive protein in preterm very low birthweight infants. Arch Dis Child Fetal Neonatal Ed. 1997;77(3):F221-F227.

39. Ng PC, et al. Quantitative measurement of monocyte HLA-DR expression in the identification of early-onset neonatal infection. Biol Neonate. 2006;89(2):75-81.

40. Walsh MC, Kliegman RM, Fanaroff AA. Necrotizing enterocolitis: a practitioner's prospective. Pediatr Rev. 1988;9(7):219-226.

41. Wong MY, et al. A magnetic bead-based serum proteomic fingerprinting method for parallel analytical analysis and micropreparative purification [published online ahead of print April 22, 2010]. Electrophoresis. doi:10.1002/elps.200900571.

42. Poon TC, et al. Prediction of liver fibrosis and cirrhosis in chronic hepatitis B infection by serum proteomic fingerprinting: a pilot study. Clin Chem. 2005;51(2):328-335.

43. Ward DG, et al. Preclinical and post-treatment changes in the HCC-associated serum proteome. Br J Cancer. 2006;95(10):1379-1383. 